\title{
Confronting Entrenched Insurgents
}

\author{
Edward H. Kaplan \\ Yale School of Management, Yale School of Public Health, Yale School of Engineering and Applied Science, Yale University, \\ New Haven, Connecticut 06520, edward.kaplan@yale.edu \\ Moshe Kress, Roberto Szechtman \\ Operations Research Department, Naval Postgraduate School, Monterey, California 93943 \\ \{mkress@nps.edu, rszechtm@nps.edu\}
}

\begin{abstract}
During counterinsurgency operations, government forces with superior firepower confront weaker low-signature insurgents. Under what conditions should government (Blue) forces attack insurgent (Red) strongholds? How should the government allocate its force across different strongholds when the insurgents' threat to the Blue civilian population must be taken into account? How should the government respond to "smart" insurgents who anticipate the government's optimal plan of attack and prepare accordingly? How do the results change when the government takes Red civilian casualties resulting from attacks on insurgent strongholds into account? This article addresses these questions. Using Lanchester models modified to account for imperfect intelligence, we formulate an optimal force allocation problem for the government and develop a knapsack approximation that has tight error bounds. We also model a sequential force allocation game between the insurgents and the government and solve for its equilibrium. When the government has perfect intelligence, in equilibrium the insurgents concentrate their force in a single stronghold that the government either attacks or not depending upon the resulting casualty count. Otherwise, under reasonable assumptions regarding the government's behavior and intelligence capabilities, it is optimal for the insurgents to "spread out" in a way that maximizes the number of soldiers required to win all battles. If the government worries about Red civilian casualties, the insurgents have a strong incentive to blend in with the Red civilian population, because this can prevent government attacks while allowing the insurgents to inflict casualties on Blue civilians. Such strategic behavior makes it harder for the government to protect its citizens from insurgent attacks.
\end{abstract}

Subject classifications: counterinsurgency; optimal force allocation; Lanchester models; value of intelligence; game theory; Hezbollah/Israel conflict.

Area of review: Military and Homeland Security.

History: Received April 2008; revisions received January 2009, April 2009; accepted April 2009. Published online in

Articles in Advance November 19, 2009.

\section{Introduction and Motivation}

Many recent military conflicts can be characterized as asymmetric combat situations pitting government forces against guerilla, insurgent, or terrorist organizations. In Afghanistan, U.S. forces have been fighting the Taliban and other fundamentalist groups for several years (Barno 2007); in Iraq, coalition forces are confronting both Sunni and Shiite insurgencies (Hoffman 2004, Cordesman and Davies 2007); and in Colombia, a mixture of armed leftist guerrilla groups and drug barons challenge the local government with continuous violence (Rabasa and Chalk 2001). In the summer of 2006, following the kidnapping of two Israeli soldiers and missile attacks on northern Israel, the Israel Defense Forces (IDF) launched an attack on the Hezbollah in Southern Lebanon. Although the force ratio was in favor of the Israelis by almost an order of magnitude, the outcome of this war was, at best, mixed from the Israeli perspective. There was no decisive victory (Blanford 2006, Cordesman 2007, Shelah and Limor 2007). The Hezbollah combatants, diffused and hidden in defensive positions in several villages and small towns, were elusive targets for the superior Israeli fire power. The IDF troops had the military means and capabilities to effectively engage the Hezbollah targets, but simply could not find most of them. Although inflicting heavy casualties on Hezbollah, the IDF forces were unsuccessful in capturing the villages, and this failure to eliminate the guerrilla units resulted in a continuous barrage of missiles on northern Israel. This example reflects the key advantage of insurgent forces, namely, their elusiveness and low-target signature, which facilitate effective "strike-and-hide" tactics.

To model specific battles between government (Blue) troops and insurgents (Red), we employ Lanchester-style models (Lanchester 1916), where individual battles are described by sets of differential equations. Lanchester models have been used in the past to describe guerrilla warfare. Deitchman (1962) presented the first such model, which was followed by Schaffer (1968). The engagement dynamics in these papers are asymmetric and involve a mixture of precise (aimed) and imprecise (area) fire: Guerrillas can observe the movement and location of government forces and engage with aimed fire, whereas government forces have limited situational awareness and therefore engage with area fire. Aimed fire leads to the Lanchester Square 
Law, whereas area fire leads to the Lanchester Linear Law (Morse and Kimball 1946).

There are very few models addressing the role of intelligence in general combat, and none in the context of guerrilla or counterinsurgency warfare. A first attempt to incorporate the effect of situational awareness, command and control, and intelligence in a Lanchester model is reported by Schreiber (1964), where these capabilities refer to the ability to distinguish between surviving and killed targets. The effect of intelligence on the distribution and specific types of air-to-air, ground-to-air, and air-to ground engagements is discussed in Allen (1993).

The Lanchester models in this paper extend Deitchman's (1962) approach in two ways. First, we incorporate an explicit intelligence function that manifests the targeting capabilities of government forces against the insurgents. Second, we employ a damage function that accounts for Blue civilian casualties inflicted by the insurgents in addition to government force battle casualties, and later extend the analysis to include Red civilian casualties.

In this paper, we model government attacks on insurgent strongholds. We use the term stronghold for a village or town in which armed insurgents are present. We develop models for optimally allocating government forces to attack insurgents dispersed in several strongholds to minimize the total number of casualties (Blue civilian and soldiers) caused by the insurgents. We also use game theory to model "smart" insurgents who are able to anticipate optimal government countertactics and deploy in a manner that leads to worst-case results for the government, and extend our results to incorporate the impact of collateral Red civilian casualties on government decision making.

From the government's standpoint, we make a distinction between high- and low-level intelligence. Reconnaissance, satellite/aerial imagery, informants, and experience from past battles regarding likely weaponry, force sizes, and topography are all viable approaches to gaining high-level intelligence regarding the allocation of insurgent forces and their capabilities; thus, we grant the government perfect high-level intelligence. Low-level intelligence governing the precise locations of insurgent fighters within strongholds is modeled via the introduction of intelligence functions that dictate the probability of successfully neutralizing insurgents given that they are fired upon. From the insurgents' standpoint, government preparations and troop movements are often directly observed; thus, we grant the insurgents perfect intelligence at both high and low levels. Even if the insurgents cannot ascertain everything regarding the deployment of government troops, modeling insurgent behavior as stemming from perfect high- and low-level intelligence presents a worst-case scenario for the government, which is what we are after.

We show that if the government has perfect low-level intelligence, in equilibrium the insurgents concentrate all of their force in a single stronghold that the government either attacks or not depending upon the resulting casualty count. Otherwise, under reasonable assumptions regarding the government's low-level intelligence capabilities and behavior, it is optimal for the insurgents to "spread out" and induce government attacks on multiple strongholds to maximize the battle and Blue civilian casualties the insurgents can inflict. In addition, if the government worries about collateral Red civilian casualties, the insurgents have a strong incentive to blend in with the Red civilian population because this can prevent government attacks while allowing the insurgents to inflict Blue civilian casualties. Not surprisingly, improved intelligence and greater total force are the main levers by which the government can reduce total casualties.

This paper is organized proceeds as follows: In the next section, we develop the intelligence-dependent Lanchester model and define the associated damage function. In $\S 3$, we consider the force allocation problem when the government knows how many insurgents are present in each stronghold, but does not know insurgents' locations within strongholds with certainty. We develop an easy-to-implement knapsack allocation policy for the government, illustrate this model in a symmetric case that yields closed-form solutions for insight, and consider a more realistic "Towns and Villages" example based on the 2006 Lebanon war. In \$4, we study a sequential game that ensues when the insurgents anticipate government actions and plan accordingly. We show that if the government follows the knapsack policy of $\S 3$, the insurgents can prepare by allocating their fighters across strongholds to maximize the number of soldiers required to win all battles, and inducing the government to select a poor knapsack sequence. We illustrate this game by building on our earlier example. Section 5 introduces Red civilian casualties caused by government attacks on insurgent strongholds under the assumptions that although the government seeks to avoid such casualties, the insurgents do not care. We show how these assumptions change our earlier results, and in particular show that when the government seeks to avoid Red civilian casualties associated with attacks on insurgent strongholds, the insurgents have an incentive to blend in with the civilian population. Section 6 presents concluding remarks. Mathematical proofs are given in the electronic companion, which is part of the online version that can be found at http://or.journal.informs.org/.

\section{Lanchester Battles and Damage Function}

Consider a government force of size $x_{0}$ that engages a stronghold held by $y_{0}$ insurgents. The insurgents have perfect information regarding the location and movement of the government force and therefore engage with aimed fire. The effectiveness of the government force, however, is limited by the intelligence governing the precise locations of insurgents within the stronghold. Let $x(t)$ and $y(t)$ denote the surviving number of government soldiers and 
the insurgents, respectively, at time $t$. We model this situation using Lanchester-style equations modified to account for the asymmetric nature of counterinsurgency operations. Specifically,

$\frac{d x(t)}{d t}=-\beta y(t)$

and

$\frac{d y(t)}{d t}=-\alpha x(t) p(y(t))$

where $\alpha$ and $\beta$ are the attrition rates of insurgents and soldiers, respectively. The intelligence function $p(y)$ models the per-shot probability of targeting an insurgent. This function depends on the degree of intelligence the soldiers possess regarding insurgent deployment as well as the number of surviving insurgents at the time of fire. We will discuss a specific form of $p(y)$ later on, but first we present more general results.

We presume for simplicity that the battle ends when one side eliminates all those on the other. ${ }^{1}$ The analysis proceeds in standard fashion by noting that

$\delta(t)=\frac{\alpha}{2} x^{2}(t)-\beta \int_{0}^{y(t)} \frac{u}{p(u)} d u$

equals a constant with respect to time, say $\delta$, for from (1)-(2),

$$
\frac{d \delta(t)}{d t}=\alpha x(t) \frac{d x(t)}{d t}-\beta \frac{y(t)}{p(y(t))} \frac{d y(t)}{d t}=0 .
$$

Equation (3) thus defines a trajectory in the $x, y$ phase plane relating the two dynamic variables $x(t)$ and $y(t)$ to each other at all times. Equation (3) must also hold at time 0, which implies that

$\delta(0) \equiv \delta=\frac{\alpha}{2} x_{0}^{2}-\beta \int_{0}^{y_{0}} \frac{u}{p(u)} d u$.

The government soldiers are victorious if the insurgents are vanquished leaving a positive number of surviving government troops. This can only occur if $\delta>0$, and from Equation (5), this criterion for government victory over the insurgents is equivalent to

$x_{0}>\sqrt{\frac{2 \beta}{\alpha} \int_{0}^{y_{0}} \frac{u}{p(u)} d u} \equiv B$,

where $B$ is the victory threshold-the minimum number of soldiers needed to defeat the $y_{0}$ insurgents. Via Equations (3)-(5), when the government wins the surviving number of soldiers, $x_{s}$ satisfies

$\frac{\alpha}{2} x_{s}^{2}-0=\delta=\frac{\alpha}{2}\left(x_{0}^{2}-B^{2}\right)$,

which yields

$x_{s}=\sqrt{x_{0}^{2}-B^{2}}$,

while $x_{0}-x_{s}$ soldiers fall in the battle.
Suppose that if the soldiers are not victorious, or if the government chooses not to attack the stronghold, the insurgents cause $k$ civilian casualties. For example, as in the Second Lebanon War between Hezbollah and Israel, the stronghold could be the source of missiles fired upon the general population that would cause damage equivalent to losing $k$ civilian lives. Or, the stronghold could be a base from which insurgents initiate an improvised explosive device (IED), suicide bombing, or other attacks targeting civilians. Whether it is worthwhile to attack the insurgents no longer depends solely upon whether or not victory can be achieved but also on the total number of casualties-civilians and soldiers. We recognize that the damage insurgents can inflict is not strictly binary depending upon whether their stronghold freely operates or is destroyed, but given that the potential for harming civilians is so much greater in the first situation than the second, we treat insurgent-inflicted civilian casualties as the fixed constant $k$ if a stronghold is not conquered, and zero otherwise. With equivalent valuation of civilians and soldiers, ${ }^{2}$ and utilizing (8), the damage function representing the total number of casualties as a function of the size of the government force $x_{0}$ is given by

$d\left(x_{0}\right)= \begin{cases}k+x_{0}, & x_{0} \leqslant B, \\ x_{0}-\sqrt{x_{0}^{2}-B^{2}}, & x_{0}>B .\end{cases}$

A plot of $d\left(x_{0}\right)$ with $B=100$ and $k=50,100$, and 150 appears in Figure 1.

To ensure that fewer battle casualties result from a successful attack on the insurgent stronghold than the number of civilian casualties that would occur in the absence of such an attack, that is, $d\left(x_{0}\right)<k$, the size of the attacking force must be sufficiently large. This condition is satisfied when

$x_{0}> \begin{cases}\frac{k}{2}+\frac{B^{2}}{2 k}, & k \leqslant B, \\ B, & k>B .\end{cases}$

Figure 1. Damage function.

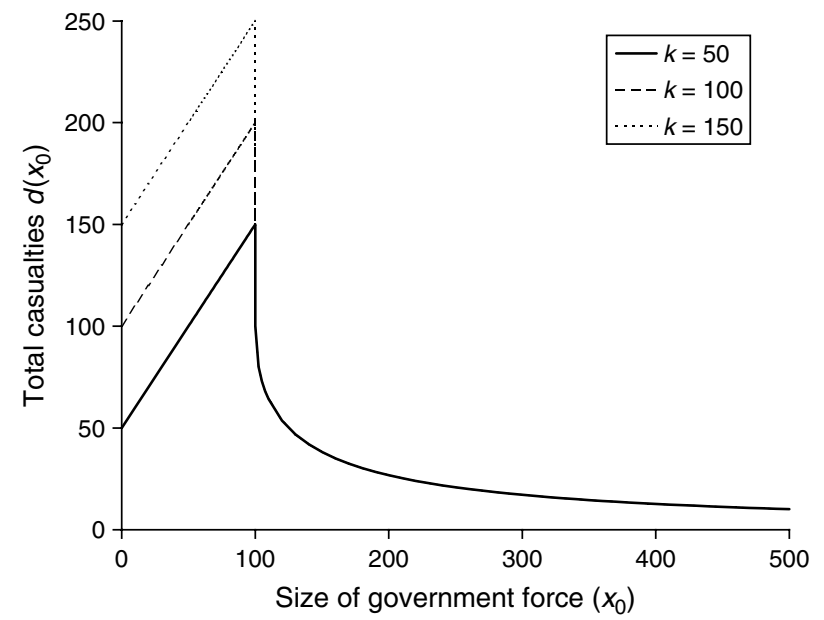


Inequality (10) shows that the minimum size of the attacking force required to ensure that $d\left(x_{0}\right)<k$ is a decreasing function of $k$ for $k \leqslant B$, and constant thereafter. This argues against attacking insurgents where the benefits of success are $\operatorname{sim}(k$ is small) even if the ability to succeed is apparent (at least $B$ soldiers can be deployed in the attack), for absent overwhelming force, the number of soldiers lost in combat could exceed the downstream casualties averted by defeating the insurgents. Note that in Figure 1, the minimum troops to be allocated equals 100 when $k=100$ and 150 , but 125 when $k=50$ in accord with inequality (10).

\section{Optimal Force Allocation}

Suppose that the insurgents are dispersed in $m$ strongholds, where the $i$ th stronghold is defended by $y_{i}$ insurgents, and conquering it would avert $k_{i}$ civilian casualties. The government has two decisions to make: which (if any) strongholds to attack, and how many soldiers $x_{i}$ to allocate to each stronghold $i$ pursued. Whereas in regular warfare forces operate in squads, companies etc., counterinsurgency operations require a more flexible force structure that can be tailored to a specific threat. We therefore assume that $x_{i}$ can assume any integer value. We also assume for simplicity that all $x_{i}$ s are determined simultaneously (the actual attacks need not occur simultaneously, providing that neither the insurgents nor the government have any recourse once the insurgent and government forces have been allocated). In addition to the force sizes $x_{i}$ and $y_{i}$, the battle conditions in the $i$ th stronghold are determined by the attrition rates $\alpha_{i}$ and $\beta_{i}$ and by the intelligence function $p_{i}(y)$. Recall that the latter four parameters determine the victory threshold $B_{i}$ (Equation (6)). The government has a total force of size $f$ to allocate to battles in the various strongholds. To keep the notation simple, without loss of generality, we assume for the remainder of this article that

$\frac{k_{1}}{B_{1}} \geqslant \frac{k_{2}}{B_{2}} \geqslant \cdots \geqslant \frac{k_{m}}{B_{m}}$.

As a step towards determining the optimal force allocation, we initially assume that the set of strongholds to be engaged, $V$, is given, and that $f$ is sufficiently large to defeat all insurgent strongholds in that set (we will relax these assumptions shortly). Because all strongholds in $V$ are defeated, there are no civilian casualties emanating from those strongholds; thus, minimizing the total number of casualties is equivalent to maximizing the number of surviving soldiers. The latter is determined from Equation (8) for each battle, which leads to the following optimization problem:

$$
\begin{aligned}
\max _{x} & \sum_{i \in V} \sqrt{x_{i}^{2}-B_{i}^{2}} \\
\text { s.t. } & \sum_{i \in V} x_{i}=f, \quad x_{i} \geqslant 0 .
\end{aligned}
$$

The solution to this problem, which is easily found by placing a Lagrange multiplier on the total force constraint and differentiating, is given by

$x_{i}^{*}=\frac{B_{i}}{\sum_{j \in V} B_{j}} f \quad$ for $i \in V$.

Equation (14) shows that given a set of strongholds to engage, the optimal allocation of soldiers is proportional to $B_{i}$; the higher the victory threshold, the greater the number of soldiers allocated to that battle. In particular, the higher the quality of tactical intelligence in a certain stronghold, the fewer soldiers are allocated. We have thus reduced the problem to determining the set $V$. To do so, let the binary variables $V_{i}=1$ if stronghold $i$ is attacked, $V_{i}=0$ otherwise, and consider the following optimization problem:

$$
\begin{array}{ll}
\min _{V, x} & \sum_{i=1}^{m} k_{i}\left(1-V_{i}\right)+\sum_{i=1}^{m}\left(x_{i}-\sqrt{x_{i}^{2}-B_{i}^{2}}\right) V_{i} \\
\text { s.t. } & x_{i} \sum_{j=1}^{m} B_{j} V_{j}=B_{i} f V_{i} \quad \text { for } i=1,2, \ldots, m, \\
& V_{i} \in\{0,1\} \quad \text { for } i=1,2, \ldots, m .
\end{array}
$$

The objective function (15) is the total damage (see (9)) across all battles. Constraint (16) ensures that if the $i$ th stronghold is attacked, then the number of soldiers allocated to that battle will follow (14), whereas if the $i$ th stronghold is not attacked, then no soldiers are allocated there. The constraint set being compact ensures that there exists (at least) an optimal solution, $V^{*}=\left(V_{1}^{*}, \ldots, V_{m}^{*}\right)$.

Because $\sum_{i=1}^{m} k_{i}$ is a constant, the preceding objective function can be framed in terms of maximizing casualties averted, $\sum_{i=1}^{m}\left(k_{i}-x_{i}+\sqrt{x_{i}^{2}-B_{i}^{2}}\right) V_{i}$. Enforcing Equation (14) leads, after elementary algebra, to the equivalent optimization problem:

$$
\begin{aligned}
\max _{V} & \sum_{i=1}^{m} k_{i} V_{i}-f+\sqrt{f^{2}-\left(\sum_{i=1}^{m} B_{i} V_{i}\right)^{2}} \\
\text { s.t. } & \sum_{j=1}^{m} B_{j} V_{j} \leqslant f, \\
& V_{i} \in\{0,1\} \quad \text { for } i=1,2, \ldots, m .
\end{aligned}
$$

\subsection{Knapsack Approximation to Optimal Force Allocation}

Although the formulation (15)-(17) above can be employed to determine optimal force allocations in any particular instance, we can develop greater insight with an approximation that yields analytical results. In the optimal solution, soldiers are allocated to battles within the optimal set $V$ in accordance with Equation (14); here we develop a fast method for approximating the optimal battle set.

Let

$K=\frac{f}{\sum_{j \in V} B_{j}}$ 
and write

$x_{i}^{*}= \begin{cases}K B_{i}, & i \in V, \\ 0, & i \notin V .\end{cases}$

$K$ is the multiple of the minimum number of soldiers necessary to conquer any stronghold in the optimal solution within the battle set $V$ (see Equation (14)). Consider the following integer knapsack conditional on $K$ (assume that the problem is feasible; shortly we will restrict $K$ to a set of values that guarantees feasibility):

$$
\begin{aligned}
\max _{V} & \sum_{i=1}^{m}\left\{k_{i}-K B_{i}+\sqrt{\left(K B_{i}\right)^{2}-B_{i}^{2}}\right\} V_{i} \\
\text { s.t. } & K \sum_{i=1}^{m} B_{i} V_{i}=f, \\
& V_{i} \in\{0,1\} \text { for } i=1,2, \ldots, m .
\end{aligned}
$$

The objective function (23) is derived from (15), (21), and (22), and it represents the total casualties averted by attacking the insurgents. Constraint (24) defines the relation between $K$ and the $V_{i}$ variables.

Rather than solving (23)-(25) exactly, we approximate as follows: Consider the ratio

$$
\begin{aligned}
r_{i} & =\frac{k_{i}-K B_{i}+\sqrt{\left(K B_{i}\right)^{2}-B_{i}^{2}}}{K B_{i}} \\
& =\frac{k_{i}}{K B_{i}}-1+\frac{\sqrt{K^{2}-1}}{K},
\end{aligned}
$$

and note that $r_{i}>r_{j}$ if and only if $k_{i} / B_{i}>k_{j} / B_{j}$. For a given $K$, an approximate solution to problem (23)-(25) is found simply by rank ordering the targets from largest to smallest $r_{i}$, and choosing the first $j^{*}(K)$ battles in this ordering to fight, where $j^{*}(K)$ is the largest value of $j$ such that $\sum_{i=1}^{j^{*}(K)} K B_{i} \leqslant f$ within the ranking (shortly we will see that the government will always use all available force if one or more strongholds are attacked). The optimal force allocations for this conditional (on $K$ ) knapsack are simply

$x_{i}^{*}(K)= \begin{cases}K B_{i}, & i \leqslant j^{*}(K), \\ 0, & i>j^{*}(K) .\end{cases}$

(We will only consider values of $K$ such that (27) is true.)

To find the optimal value of $K$, we will only consider values of $K$ that exhaust the force with equality because the entire force will be expended in the optimal solution. Let $K_{j}$ correspond to that value of $K$ that would allocate soldiers to the first $j$ battles within the knapsack ranking. From the force allocation constraint, we have

$K_{j}=\frac{f}{\sum_{h=1}^{j} B_{h}} \quad$ for $j=1,2, \ldots, m$ with $K_{0} \equiv 0$. When $K=K_{j}$, Equation (27) becomes

$x_{i}^{*}\left(K_{j}\right)= \begin{cases}\frac{B_{i}}{\sum_{h=1}^{j} B_{h}} f, & i \leqslant j, \\ 0, & i>j,\end{cases}$

with $x_{i}^{*}(0)=K_{0} B_{i}=0$ (so no strongholds are attacked when $K=K_{0}=0$ ).

Now, revisit Equation (23) after replacing $m$ by $j, K$ by $K_{j}$ (using Equation (28)), and setting $V_{i}=1, i=1,2, \ldots, j$ to obtain

$\max _{0 \leqslant j \leqslant j^{+}} \sum_{i=1}^{j} k_{i}-f+\sqrt{f^{2}-\left(\sum_{i=1}^{j} B_{i}\right)^{2}}$,

where $j^{+}$is the largest value of $j$ such that $\sum_{i=1}^{j} B_{i} \leqslant f$, and $j=0$ corresponds to the decision not to fight any battles (in which case no casualties are averted). This problem is easily solved by enumeration over $j$. Let $j^{*} \in\left\{0,1, \ldots, j^{+}\right\}$ be the smallest number that maximizes (30). The knapsackoptimal allocation is given by

$x_{i}^{*}= \begin{cases}\frac{B_{i}}{\sum_{h=1}^{j^{*}} B_{h}} f, & i \leqslant j^{*}, \\ 0, & i>j^{*} .\end{cases}$

Let us consider the error incurred by the knapsack approximation. Clearly,

$$
\begin{array}{r}
\sum_{i=1}^{m} k_{i} V_{i}^{*}-f+\sqrt{f^{2}-\left(\sum_{i=1}^{m} B_{i} V_{i}^{*}\right)^{2}} \\
\geqslant \sum_{i=1}^{j^{*}} k_{i}-f+\sqrt{f^{2}-\left(\sum_{i=1}^{j^{*}} B_{i}\right)^{2}}
\end{array}
$$

if $j^{*}>0$, and

$\sum_{i=1}^{m} k_{i} V_{i}^{*}-f+\sqrt{f^{2}-\left(\sum_{i=1}^{m} B_{i} V_{i}^{*}\right)^{2}} \geqslant 0$

if $j^{*}=0$. We can upper bound the left-hand side above by its integer relaxation:

$$
\begin{gathered}
\max _{U \in \mathscr{M}_{f}} \sum_{i=1}^{m} k_{i} U_{i}-f+\sqrt{f^{2}-\left(\sum_{i=1}^{m} B_{i} U_{i}\right)^{2}} \\
\geqslant \sum_{i=1}^{m} k_{i} V_{i}^{*}-f+\sqrt{f^{2}-\left(\sum_{i=1}^{m} B_{i} V_{i}^{*}\right)^{2}},
\end{gathered}
$$

where $M_{f}=\left\{U_{i} \in[0,1]: \sum_{i=1}^{m} B_{i} U_{i} \leqslant f\right\}$. Let $U^{*}=$ $\left(U_{1}^{*}, \ldots, U_{m}^{*}\right)$ be a solution to the integer relaxation problem. The next proposition relates $U^{*}$ to the knapsack approximation solution (all proofs appear in the online appendix). 
Proposition 1. Suppose that inequality (11) holds. Then,

(i) If $f<B_{1}\left(1+\left(B_{1} / k_{1}\right)^{2}\right)^{1 / 2}$, then $U_{1}^{*} \in(0,1)$ and $U_{i}^{*}=0$ for $i=2, \ldots, m$. Also, $j^{*}$ equals 0 or 1 .

(ii) If $\left(\sum_{i=1}^{\nu} B_{i}\right)\left(1+\left(B_{\nu+1} / k_{\nu+1}\right)^{2}\right)^{1 / 2}<f<\left(\sum_{i=1}^{\nu+1} B_{i}\right)$. $\left(1+\left(B_{\nu+1} / k_{\nu+1}\right)^{2}\right)^{1 / 2}$ for some $\nu \in\{1, \ldots, m-1\}$, then $U_{i}^{*}=1$ for $i=1, \ldots, \nu, U_{\nu+1}^{*} \in(0,1)$, and $U_{i}^{*}=0$ for $i=$ $\nu+2, \ldots, m$. Also, $j^{*}$ equals $\nu$ or $\nu+1$.

(iii) If $\left(\sum_{i=1}^{\nu} B_{i}\right)\left(1+\left(B_{\nu} / k_{\nu}\right)^{2}\right)^{1 / 2} \leqslant f \leqslant\left(\sum_{i=1}^{\nu} B_{i}\right)$. $\left(1+\left(B_{\nu+1} / k_{\nu+1}\right)^{2}\right)^{1 / 2}$ for some $\nu \in\{1, \ldots, m-1\}$, then $U_{i}^{*}=1$ for $i=1, \ldots, \nu, U_{i}^{*}=0$ for $i=\nu+1, \ldots, m$, and $j^{*}=\nu$.

(iv) If the force level $f$ is sufficiently large, $f \geqslant$ $\left(\sum_{i=1}^{m} B_{i}\right)\left(1+\left(B_{m} / k_{m}\right)^{2}\right)^{1 / 2}$, then $U_{i}^{*}=1$ for $i=1, \ldots, m$, and $j^{*}=m$.

Recall that the solution to problem (18)-(20) is sandwiched between its integer relaxation (above) and the knapsack approximation (below). Hence, in cases (iii) and (iv) of Proposition 1, the number of casualties averted produced by the knapsack approximation coincides with the optimal number of casualties averted, obtained by solving problem (18)-(20). In case (ii), Proposition 1 shows that the difference in number of casualties averted is bounded above:

$$
\left.\begin{array}{rl}
\left(\sum_{i=1}^{m} k_{i} V_{i}^{*}-f+\sqrt{f^{2}-\left(\sum_{i=1}^{m} B_{i} V_{i}^{*}\right)^{2}}\right) & \\
& -\left(\sum_{i=1}^{j^{*}} k_{i}-f+\sqrt{f^{2}-\left(\sum_{i=1}^{j^{*}} B_{i}\right)^{2}}\right) \\
\leqslant & \left(\sum_{i=1}^{m} k_{i} U_{i}^{*}-f+\sqrt{f^{2}-\left(\sum_{i=1}^{m} B_{i} U_{i}^{*}\right)^{2}}\right) \\
& -\left(\sum_{i=1}^{j^{*}} k_{i}-f+\sqrt{f^{2}-\left(\sum_{i=1}^{j^{*}} B_{i}\right)^{2}}\right)^{2} \\
= & k_{\nu+1} U_{\nu+1}^{*}+\sqrt{f^{2}-\left(\sum_{i=1}^{\nu} B_{i}+B_{\nu+1} U_{\nu+1}^{*}\right)^{2}} \\
& -\max \left\{\sqrt{f^{2}-\left(\sum_{i=1}^{\nu} B_{i}\right)^{2}}, k_{\nu+1}+\sqrt{f^{2}-\left(\sum_{i=1}^{\nu+1} B_{i}\right)^{2}}\right.
\end{array}\right\}
$$

The above argument remains valid for case (i) of Proposition 1 , with $\nu=0$. In conclusion, the number of casualties averted obtained by the knapsack approximation may be lower than the optimal number of casualties averted, but the difference is smaller than $k_{\nu+1}$.

\subsection{Example 1: Symmetric Insurgents$$
\left(k_{i}=k, B_{i}=B\right)
$$

Consider the special case where $k_{i}=k$ and $B_{i}=B$ for all battles. Then, the objective in (30) becomes

$$
\max _{j} j k-f+\sqrt{f^{2}-j^{2} B^{2}}
$$

Treating (32) as a continuous optimization problem, we obtain that the optimal number of strongholds to attack is

$j^{*}=\sqrt{\frac{k^{2}}{B^{2}+k^{2}}} \frac{f}{B}$,

which is assured to be smaller than the maximum number of battles that could be fought, $j^{+}=f / B$ (ignoring integrality). The optimal number of soldiers allocated per battle fought equals

$x^{*}=\frac{f}{j^{*}}=\sqrt{\frac{B^{2}+k^{2}}{k^{2}}} B$.

Total casualties averted are found by substituting $j^{*}$ into the objective function (32) and equal

casualties averted $=\left(\sqrt{\frac{B^{2}+k^{2}}{B^{2}}}-1\right) f$.

These results accord with intuition: The optimal number of battles attenuates the maximum number of battles that could be fought $(f / B)$ by the factor $\sqrt{k^{2} /\left(B^{2}+k^{2}\right)}$. If $k$ is very large relative to $B$, this factor tends towards one, and it is optimal to fight as many battles as possible to avert a large number of civilian casualties. If $k$ is very small relative to $B$, then it is not optimal to fight any battles $\left(j^{*} \rightarrow 0\right)$ because battle casualties would exceed the downstream casualties averted.

\subsection{Example 2: Insurgents in Towns and Villages}

Consider an attack on a town where $y$ insurgents grouped in cells of $c$ insurgents per cell are dispersed among $l$ distinct sites (houses, buildings) within the town. There are $y / c$ different cells spread amongst the $l$ sites; thus, the probability that a randomly selected structure contains insurgents is equal to $(y / c) / l=y / n$, where $n=c l$.

This example is based on general and partial data regarding the 2006 war between Israel and Hezbollah in Southern Lebanon. A typical village in southern Lebanon (e.g. Maroun-A Ras, Yaroun) contains 100-200 houses and buildings, whereas within a small town (e.g., Bint Jbail, Tyre), 800-1,500 structures are typical (Google Earth 2008). We consider attacking up to five insurgent strongholds (three villages and two towns) where initially the insurgents operate in cells of size $c=10$. Left to their own devices, insurgents in the $i$ th stronghold are capable of inflicting $k_{i}$ civilian casualties. The data regarding these five strongholds are summarized in Table 1.

Next, we specify the intelligence function $p(y)$. Absent intelligence, the soldiers fire at random (area fire); thus, only a fraction $y / n$ of their fire is effective. In this (worst) case, $p(y)=y / n$, and from (2) we obtain the Deitchman model with

$B=\sqrt{\frac{2 \beta n y}{\alpha}}$ 
Table 1. Insurgency data: Towns and villages.

\begin{tabular}{lccc}
\hline Stronghold $(i)$ & $\begin{array}{c}\text { Insurgent } \\
\text { locations }\left(n_{i}\right)\end{array}$ & $\begin{array}{c}\text { Insurgents } \\
\left(y_{i}\right)\end{array}$ & $\begin{array}{c}\text { Civilian } \\
\text { casualties }\left(k_{i}\right)\end{array}$ \\
\hline Town 1 & 8,000 & 200 & 200 \\
Town 2 & 10,000 & 300 & 200 \\
Village 1 & 2,000 & 100 & 50 \\
Village 2 & 1,500 & 100 & 50 \\
Village 3 & 1,000 & 50 & 20 \\
\hline
\end{tabular}

(Deichtman 1962). At the other extreme, if the soldiers have perfect intelligence, then $p(y)=1$ and one obtains the Lanchester direct-fire model with

$B=\sqrt{\frac{\beta}{\alpha}} y$.

Note that the constant imperfect intelligence function $p(y)=p<1$ can always be modeled as perfect intelligence with $p(y)=1$ by reducing the government's effective fire rate from $\alpha$ to $\alpha p$. Because it is always possible to fire at random, we assume that $y / n \leqslant p(y) \leqslant 1$. As an intermediate formulation, we use a linear function (Kress and Szechtman 2009)

$p(y)=\mu+(1-\mu) \frac{y}{n}$.

This function has an intuitive interpretation: The soldiers know the location of a fraction $\mu$ of the insurgents and therefore engage them by direct fire, whereas the remaining fraction $(1-\mu)$, whose locations are not known, are engaged by area fire. Note that $\mu=0$ replicates the Deichtman model whereas $\mu=1$ replicates the original Lanchester model. Via Equation (6), the threshold value $B$ for this linear intelligence function is given by

$B=\sqrt{\frac{2 \beta n}{\alpha(1-\mu)}\left[y-\frac{n \mu}{(1-\mu)} \log \left(\frac{(1-\mu) y}{\mu n}+1\right)\right]}$.

We assume that the effectiveness ratio $\beta / \alpha$ equals 0.5 for all five strongholds attacked, which means that the (nominal) attrition rate of the insurgents (by the soldiers) is twice the attrition rate of the soldiers. Table 2 reports the maximal casualties averted and optimal soldiers allocations to the five different battles as a function of the intelligence parameter $\mu$ when the total government force is constrained to $f=$ 2,000 (about two brigades). Note that for any value of $\mu$, ranking the strongholds from largest to smallest ratio of $k / B$ yields the ordering: Town 1, Town 2, Village 2, Village 1, and Village 3. In this example, the knapsack approximation of Equations (30)-(31) yields the optimal solution to model (15)-(17). When $\mu=0$ (no tactical intelligence) the optimal strategy is attack Town 1 with full force while forfeiting Town 2 and the villages to the insurgents; this serves to prevent only 12 soldiers and civilian casualties in total, but is the best result possible. As $\mu$ increases, and tactical intelligence improves, the government is able to engage more of
Table 2. Intelligence-dependent optimal force allocations and casualties averted.

\begin{tabular}{|c|c|c|c|c|c|c|}
\hline$\mu$ & $\begin{array}{c}\text { Casualties } \\
\text { averted }\end{array}$ & Town 1 & Town 2 & Village 1 & Village 2 & Village 3 \\
\hline 0.00 & 12.14 & $2,000.00$ & 0.00 & 0.00 & 0.00 & 0.00 \\
\hline 0.05 & 121.94 & $2,000.00$ & 0.00 & 0.00 & 0.00 & 0.00 \\
\hline 0.10 & 155.94 & $2,000.00$ & 0.00 & 0.00 & 0.00 & 0.00 \\
\hline 0.15 & 201.52 & 804.02 & $1,195.98$ & 0.00 & 0.00 & 0.00 \\
\hline 0.20 & 248.80 & 802.93 & $1,197.07$ & 0.00 & 0.00 & 0.00 \\
\hline 0.25 & 277.85 & 802.25 & $1,197.75$ & 0.00 & 0.00 & 0.00 \\
\hline 0.30 & 302.06 & 671.19 & $1,003.07$ & 0.00 & 325.74 & 0.00 \\
\hline 0.35 & 324.85 & 575.31 & 860.41 & 283.48 & 280.80 & 0.00 \\
\hline 0.40 & 346.34 & 574.60 & 859.82 & 283.89 & 281.69 & 0.00 \\
\hline 0.45 & 363.12 & 574.03 & 859.34 & 284.22 & 282.41 & 0.00 \\
\hline 0.50 & 377.73 & 535.49 & 801.93 & 265.59 & 264.19 & 132.80 \\
\hline 0.55 & 390.52 & 535.11 & 801.59 & 265.78 & 264.62 & 132.89 \\
\hline 0.60 & 401.20 & 534.79 & 801.31 & 265.94 & 264.99 & 132.97 \\
\hline 0.65 & 410.25 & 534.51 & 801.06 & 266.08 & 265.31 & 133.04 \\
\hline 0.70 & 418.02 & 534.28 & 800.85 & 266.20 & 265.58 & 133.10 \\
\hline 0.75 & 424.76 & 534.07 & 800.66 & 266.30 & 265.82 & 133.15 \\
\hline 0.80 & 430.66 & 533.89 & 800.50 & 266.39 & 266.03 & 133.20 \\
\hline 0.85 & 435.87 & 533.72 & 800.35 & 266.47 & 266.21 & 133.24 \\
\hline 0.90 & 440.51 & 533.58 & 800.22 & 266.54 & 266.38 & 133.27 \\
\hline 0.95 & 444.67 & 533.45 & 800.11 & 266.61 & 266.53 & 133.30 \\
\hline 1.00 & 448.41 & 533.33 & 800.00 & 266.67 & 266.67 & 133.33 \\
\hline
\end{tabular}

the insurgent strongholds; indeed, once $\mu$ reaches 0.5 , all strongholds are attacked. The results also indicate an astonishing initial return in casualties averted to intelligencesimply knowing where 5\% of the insurgents are enables a tenfold increase in the number of casualties prevented compared to no intelligence (from 12 to 122). Diminishing returns set in thereafter, showing that although minimal intelligence is greatly preferred to none, perfect intelligence only leads to a $10 \%$ improvement in casualties averted compared to "reasonable" intelligence (e.g., $\mu=0.65$ ).

Figure 2 reports the maximal number of casualties averted and optimal force allocations across the five insurgent strongholds. We vary the overall size of the attacking force $f$ from 0 to 2,000, while holding all other parameters constant at their earlier values and fixing $\mu=0.5$. Figure 2 shows the scalloping, piecewise-concave nature of the optimal casualties averted function while reporting the split of the total force in proportion to those $B_{i} \mathrm{~s}$ in the optimal battle set $V$. The sharpest growth in casualties averted occurs when $199 \leqslant f \leqslant 546$ and only Town 1 is attacked. At small total force levels $(73 \leqslant f \leqslant 145)$ only Village 3 is attacked (for $f \leqslant 72$, no strongholds are attacked because launching any strikes would increase total casualties beyond $\sum_{i} k_{i}=520$ ), whereas for $146 \leqslant f \leqslant 198$, only Village 2 is attacked. The knapsack approximation produces optimal results for all values of $f \geqslant 670$, but for smaller values of $f$ the optimal decisions resulting from the original force allocation model (15)-(17) depart from the knapsack values. For example, the knapsack approximation does not recommend any attacks until $f=200$, at which point Town 1 is attacked, whereas for $547 \leqslant f \leqslant 669$, when the optimal decision is to attack Town 1 and Village 2, 
Figure 2. Optimal force allocation and casualties averted.

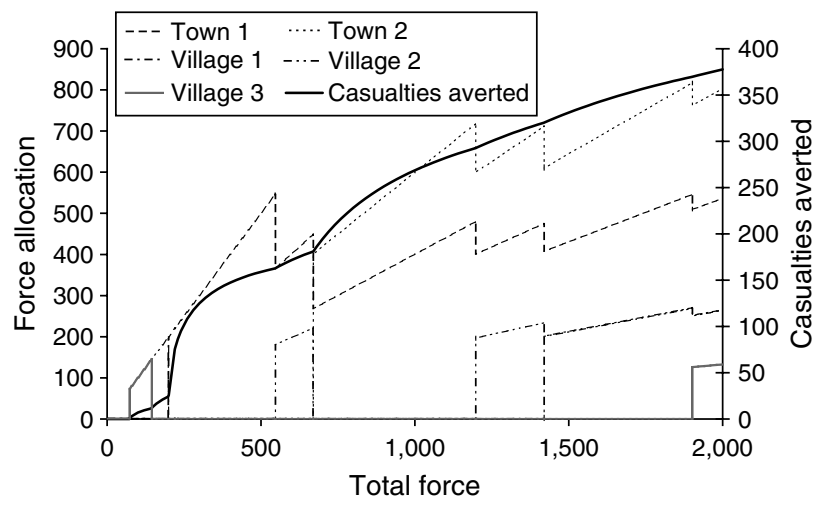

the knapsack approximation recommends attacking only Town 1 or Towns 1 and 2, depending upon the value of $f$. The largest percentage error in the total number of casualties between the optimal and knapsack results equals $4.9 \%$ when $f=198$ (the optimal strategy is to attack Village 2, but the knapsack recommends abstaining from any attacks, forgoing the opportunity to avert 24 casualties).

\section{Sequential Force Allocation Game}

So far, we have assumed that only the government faces the force allocation problem. However, if the insurgents knew about the government's situation, then they would allocate their resources (human and materiel) to inflict the largest possible amount of damage. Given the hesitance of governments to respond to insurgents unless provoked, we will model this situation as a sequential game with perfect information where the insurgents first position their fighters and materiel to inflict maximal battle and civilian casualties on the government, after which the government allocates its force to minimize the damage caused by the insurgents. Although one could model this situation as a simultaneous game, and although in reality the insurgents do not know all of the government's problem parameters (intelligence function, attrition coefficients, etc.), a zero-sum sequential game provides a worst-case scenario for the government.

Suppose that the insurgents have a total of $\psi$ fighters, which results in the force levels $\mathbf{y}=\left(y_{1}, \ldots, y_{m}\right)$ belonging to the simplex $\mathbb{M}_{\psi}=\left\{\mathbf{y} \geqslant \mathbf{0} ; \sum_{i=1}^{m} y_{i} \leqslant \psi\right\}$. Also, suppose that the insurgents may inflict at most $\kappa$ civilian casualties, meaning that $\mathbf{k}=\left(k_{1}, \ldots, k_{m}\right)$ belongs to the simplex $\mathcal{M}_{\kappa}=\left\{\mathbf{k} \geqslant \mathbf{0} ; \sum_{i=1}^{m} k_{i} \leqslant \kappa\right\}$. Faced with an insurgency spread according to certain $\mathbf{y}$ and $\mathbf{k}$, the government allocates its forces following Equation (14) and decides where to fight by solving problem (15)-(17), leading to $\eta(\mathbf{y}, \mathbf{k})$ casualties in total. Thus, the insurgents' problem is to

$$
\begin{array}{cl}
\max _{y, k} & \eta(\mathbf{y}, \mathbf{k}) \\
\text { s.t. } & \mathbf{y} \in M_{\psi}, \\
& \mathbf{k} \in M_{\kappa} .
\end{array}
$$

Clearly, $\mathbf{y}$ and $\mathbf{k}$ are such that $\sum_{i=1}^{m} y_{i}=\psi$ and $\sum_{i=1}^{m} k_{i}=\kappa$, because the insurgent's objective is making things worse for the government. Also, $k_{i}>0$ if and only if $y_{i}>0$. Indeed, $k_{i}>0$ and $y_{i}=0$ cannot be optimal because the $k_{i} \mathrm{~s}$ would be destroyed by the soldiers at zero cost, and $k_{i}=0$ and $y_{i}>0$ cannot be optimal because the government would never attack such a stronghold. Note that because the soldiers can always choose not to engage the insurgents, it follows that $\eta(\mathbf{y}, \mathbf{k}) \leqslant \kappa$ for any feasible allocation of $\mathbf{y}$ and $\mathbf{k}$. Moreover, if $f \geqslant \sum_{i=1}^{m} B_{i}\left(y_{i}\right)$ for any feasible $\mathbf{y}$, then $\eta(\mathbf{y}, \mathbf{k}) \leqslant \max _{\mathbf{y} \in M_{\psi}} \sum_{i=1}^{m} B_{i}\left(y_{i}\right)$.

The standard approach to solving problem (40)-(42) is to introduce a constraint for each feasible configuration of engagement variables $V_{1}, \ldots, V_{m}$. Unfortunately, there are $2^{m}$ nonlinear constraints and the constraint set may not be convex, which makes it impossible to obtain an analytical solution without imposing more structure on the problem.

With this in mind, we observe that a drawback of constraint (41) is that an allocation of insurgents $\mathbf{y}$ matters insofar as it impinges on the victory thresholds $B_{1}, \ldots, B_{m}$ (cf. Equation (6)). This suggests that a more natural way to express constraint (41) is via a constraint on the victory thresholds $B$. Letting $\Gamma \in R^{m}$ be the set mapped by $B_{1}\left(y_{1}\right), \ldots, B_{m}\left(y_{m}\right)$ for all $\mathbf{y} \in \mathcal{M}_{\psi}$, we have the following result.

Proposition 2. If the threshold functions $B_{1}\left(y_{1}\right), \ldots$, $B_{m}\left(y_{m}\right)$ are concave, then the set $\Gamma$ is convex.

From Equation (6), it easily follows (by taking the second derivative of $B(y))$ that convex intelligence functions $p(y)$ lead to concave threshold functions $B(y)$; this includes the linear intelligence function $p(y)=\mu+(1-\mu) y / n$ of §3.3. Indeed, all the monomials $p(y)=$ constant $\times y^{\theta}$ with $\theta \geqslant 0$ produce $B(y)$ concave. Hence, for the rest of this section we shall restrict our attention to convex sets $\Gamma$.

Looking at problem (40)-(42), the simplest scenario occurs when $p_{i}\left(y_{i}\right)=1$, that is, the government has perfect intelligence for all $i$ s. In this case, the $B_{i}(\cdot)$ functions are linear, implying that there exists an extreme pointa stronghold $i^{*}$-such that $B_{i^{*}}(\psi)=\max _{\mathbf{y} \in \mu_{\psi}} \sum_{i} B_{i}\left(y_{i}\right)$. That is, the sum of the thresholds is largest when the insurgents concentrate all their forces $\psi$ in one stronghold, $i^{*}$. In this case, there are several possible situations, depending on the relative values of $\kappa, B_{i^{*}}(\psi)$, and $f$. The results for each case are summarized in the next proposition.

Proposition 3. Suppose that $p_{i}\left(y_{i}\right)=1$ for all strongholds. Then, it is optimal for the insurgents to allocate all their resources to stronghold $i^{*}$, meaning that $y_{i^{*}}=\psi$ and $k_{i^{*}}=\kappa$, and there exist three possibilities:

(i) If $f \geqslant \kappa \geqslant B_{i^{*}}(\psi)$ or $\kappa \geqslant f \geqslant B_{i^{*}}(\psi)$, the government engages the insurgents by setting $x_{i^{*}}=f$.

(ii) If $f \geqslant B_{i^{*}}(\psi) \geqslant \kappa$, the government engages the insurgents by setting $x_{i^{*}}=f$ if and only if $f-\left(f^{2}-B_{i^{*}}^{2}(\psi)\right)^{1 / 2} \leqslant \kappa$.

(iii) If $B_{i^{*}}(\psi)>f$, the government never engages the insurgents. 
The practical implication of having $p_{i}\left(y_{i}\right)=1$ is that the insurgency is under direct government fire. In this situation, Proposition 3 asserts that the insurgents concentrate their forces and that, depending on the force level $f$ relative to $\kappa$ and $B_{i^{*}}(\psi)$, the government engages the insurgents (when battle casualties are lower than civilian casualties prevented) or not. This conclusion is consistent with the principle of force concentration, which is derived from Lanchester's Square Law (Morse and Kimball 1946).

\subsection{Knapsack Approximation Game}

In $\S 3$, we argued that problem (30) provides a very good approximation to the allocation problem (15)-(17) faced by the government. This motivates analyzing the sequential zero-sum game that emerges when the insurgency allocates its resources knowing that the government employs the knapsack approximation (30) to deploy its forces. Looking at problem (30), the insurgency minimizes casualties averted:

$$
\begin{aligned}
\min _{y, k} & \theta(\mathbf{y}, \mathbf{k}) \\
\text { s.t. } & \mathbf{y} \in \mathcal{M}_{\psi}, \\
& \sum_{i=1}^{m} k_{i}=\kappa, \quad k_{i} \geqslant 0,
\end{aligned}
$$

where

$\theta(\mathbf{y}, \mathbf{k})=\max _{0 \leqslant j \leqslant j^{+}} \sum_{i=1}^{j} k_{i}-f+\left(f^{2}-\left(\sum_{i=1}^{j} B_{i}\left(y_{i}\right)\right)^{2}\right)^{1 / 2}$.

For problem (43)-(45) to make sense, we need to write $\sum_{i=1}^{m} k_{i}=\kappa$ instead of $\sum_{i=1}^{m} k_{i} \leqslant \kappa$ in (45), because otherwise $\min _{y, k} \theta(\mathbf{y}, \mathbf{k})=0$ with $\mathbf{k}=\mathbf{0}$.

Let $j^{*}(\mathbf{y}, \mathbf{k})$ be the optimal value of $j$ when viewed as a function of $(\mathbf{y}, \mathbf{k})$, and recall that the rank-ordering assumption, Equation (11), is in force. Because the set of feasible allocations is compact, there exists an optimal solution for the insurgency, $(\tilde{\mathbf{y}}, \tilde{\mathbf{k}})$. To simplify the notation, we write $\tilde{B}_{i}=B_{i}\left(\tilde{y}_{i}\right), B_{i}=B_{i}\left(y_{i}\right)$, for $i=1, \ldots, m$.

To breach into problem (43)-(45), notice that because the government may choose to bear the civilian casualties without fighting (i.e., $j=0$ ), we have $\theta(\mathbf{y}, \mathbf{k}) \geqslant 0$. Thus, any allocation $(\mathbf{y}, \mathbf{k})$ that achieves this lower bound is optimal for the insurgents. Also, the best the government can do is to avert $\kappa$ casualties at low soldiers' casualties, so that $\theta(\mathbf{y}, \mathbf{k}) \leqslant \kappa$.

Recall that the government selects the towns to engage or not in decreasing order of the ratios $k_{i} / B_{i}$. This suggests that a judicious approach for the insurgency is to flatten the ratios as much as possible. As the next proposition demonstrates, there exist insurgent optimal solutions with $k_{i} / B_{i}$ constant.
Proposition 4. Suppose that the government allocates its forces by solving problem (30). Then, $\theta(\tilde{\mathbf{y}}, \tilde{\mathbf{k}})=\theta(\tilde{\mathbf{y}}, \hat{\mathbf{k}})$, where

$\hat{k}_{i}=\kappa \frac{\tilde{B}_{i}}{\sum_{j=1}^{m} \tilde{B}_{j}}$,

and the ratios $\hat{k}_{i} / \tilde{B}_{i}=\kappa / \sum_{j=1}^{m} \tilde{B}_{j}$ are constant.

Hence, because we can restrict attention to solutions $(\mathbf{y}, \mathbf{k})$ with constant ratios $k_{i} / B_{i}$, problem (43)-(45) becomes

$$
\begin{aligned}
\min _{y} \max _{0 \leqslant j \leqslant j^{+}} & \kappa \frac{\sum_{i=1}^{j} B_{i}}{\sum_{i=1}^{m} B_{i}}-f+\left(f^{2}-\left(\sum_{i=1}^{j} B_{i}\right)^{2}\right)^{1 / 2} \\
\text { s.t. } & \sum_{i=1}^{m} y_{i}=\psi, \quad y_{i} \geqslant 0 .
\end{aligned}
$$

It is easier to tackle (46)-(47) by considering the government's problem conditioned on $\sum_{i=1}^{m} B_{i} \equiv l$. Given $l$, let $j_{l}$ be the optimal number of strongholds engaged by the government, and let $\phi_{l}\left(\sum_{i=1}^{j} B_{i}\right)$ be the right-hand side of (46) (minus the $\min _{y} \max _{0 \leqslant j \leqslant j^{+}}$term). The square root term, when viewed as a function of $\sum_{i=1}^{j} B_{i}$, spans the northeast quadrant of a circumference of radius $f$, and so it is strictly concave decreasing in $\sum_{i=1}^{j} B_{i}$. Therefore, $j_{l}$ necessarily takes one of two values: the largest value of $j$ such that $\phi_{l}^{\prime}\left(\sum_{i=1}^{j} B_{i}\right) \geqslant 0$; or the smallest value of $j$ such that $\phi_{l}^{\prime}\left(\sum_{i=1}^{j} B_{i}\right) \leqslant 0$. More precisely, $j_{l}$ must equal either $r$ or $r+1$, where

$\sum_{i=1}^{r} B_{i} \leqslant \frac{f}{\left(1+(l / \kappa)^{2}\right)^{1 / 2}} \leqslant \sum_{i=1}^{r+1} B_{i}$

for viewed as a function of $\sum_{i=1}^{j} B_{i}, \phi_{l}\left(\sum_{i=1}^{j} B_{i}\right)$ is maximized when $\sum_{i=1}^{j} B_{i}=f /\left(1+(l / \kappa)^{2}\right)^{1 / 2}$. This leads to an upper bound for the number of casualties the government can avert: the best the government can do is set $\sum_{i=1}^{j} B_{i}=$ $f /\left(1+(l / \kappa)^{2}\right)^{1 / 2}$, whereas the insurgents can inflict at most $\kappa$ civilian casualties, which implies that

$\phi_{l}\left(\sum_{i=1}^{j} B_{i}\right) \leqslant \min \left\{f\left(\left(1+\frac{\kappa^{2}}{l^{2}}\right)^{1 / 2}-1\right), \kappa\right\}$.

From (48), the number of engagements taken by the government decreases with $l$ and increases with $\kappa$. Large values of $l / \kappa$ lead to $B_{1}>f\left(1+(l / \kappa)^{2}\right)^{-1 / 2}$, in which case the government fights at most one battle. On the other hand, small values of $l / \kappa$ lead to $j_{l}=m$ (indeed, from Equation (48), this must happen when $\left.l \leqslant f /\left(1+(l / \kappa)^{2}\right)^{1 / 2}\right)$. This reasoning indicates that the insurgents attempt to make $l$ as large as possible.

However, just maximizing $l$ does not tell the whole story. Indeed, looking at Equations (46) and (48), the insurgents' best option is to make the government indifferent to having 
$j_{l}=r$ or $j_{l}=r+1$. This means that for values of $l$ sufficiently large such that $j_{l}<m$, the optimal value of $B_{r+1}$ satisfies

$\phi_{l}\left(\sum_{i=1}^{r} B_{i}\right)=\phi_{l}\left(\sum_{i=1}^{r+1} B_{i}\right)$.

Moreover, on the space of feasible solutions where (48) holds, the optimal solution for the insurgency is the one that minimizes $\phi_{l}\left(\sum_{i=1}^{r} B_{i}\right)$ while preserving Equation (50), which is analogous to making both $l$ and $B_{r+1}$ large while preserving Equations (48)-(50).

In other words, the last battle offered by the insurgency is relatively large, and the net change in casualties averted by taking versus not taking it is zero. This happens because if the government takes the $(r+1)$ st town, then $k_{r+1}=$ $\kappa B_{r+1} / l$ extra casualties are averted, but additional soldiers' casualties are incurred because each battle is fought closer to its victory threshold. On the other hand, if the $(r+1)$ st town is not taken, then the government bears extra $k_{r+1}$ casualties, but the soldiers' casualties in the first $r$ battles are relatively lower. Hence, in this game the government would initially be offered battles where it is clearly advantageous to attack the insurgency, and then it would be offered a relatively (to the other battles) large battle with two equally unappealing outcomes: bear a large number of civilian casualties, or take many more soldiers' casualties.

Regarding the ordering of the battle thresholds offered by the insurgency, their order does not matter when the $B_{i}$ s have low variability. If the variability is large then the order of the $B_{i} \mathrm{~s}$ depends on $f /\left(1+l^{2} / \kappa^{2}\right)^{1 / 2}$. For $f /\left(1+l^{2} / \kappa^{2}\right)^{1 / 2}$ low, we have $j_{l}$ small, suggesting that the largest $B_{i} \mathrm{~s}$ would appear first. On the other hand, when $f /\left(1+l^{2} / \kappa^{2}\right)^{1 / 2}$ is large, we have $j_{l}$ large too, and the insurgents will put the $B_{i} \mathrm{~s}$ in increasing order, so that $B_{r+1}$ is large. Finally, the order does not matter when $f /\left(1+l^{2} / \kappa^{2}\right)^{1 / 2}$ is so large that $j_{l}=m$.

\subsection{Knapsack Games in Towns and Villages}

Consider again the towns and villages example of $\$ 3.3$. Rather than assuming that the government can optimize its attack, presuming that insurgent forces and downstream civilian casualties are distributed as in Table 1, we now model "smart" insurgents who anticipate that the government will allocate its force to maximize the objective in (30). We presume that the government has $f=2,000$ troops to allocate, whereas the insurgents have $\psi=750$ fighters along with materiel (e.g., missiles, IEDs) capable of causing $\kappa=520$ civilian casualties. The scenario and intelligence function are as in $\$ 3.3$. We examine the consequences of the resulting knapsack game as a function of the intelligence parameter $\mu$.

For a given problem instance, the insurgents deploy by allocating $\tilde{y}_{i}$ fighters to stronghold $i$ where the $\tilde{y}_{i}$ s jointly maximize $\sum_{j=1}^{m} B_{j}\left(y_{j}\right)\left(B_{j}\left(y_{j}\right)\right.$ is given by Equation (39)), set $\tilde{k}_{i}=\kappa B_{i}\left(\tilde{y}_{i}\right) / \sum_{j=1}^{m} B_{j}\left(\tilde{y}_{j}\right)$, and lightly perturb the $\tilde{k} \mathrm{~s}$
Figure 3. Casualties averted in knapsack games in towns and villages.

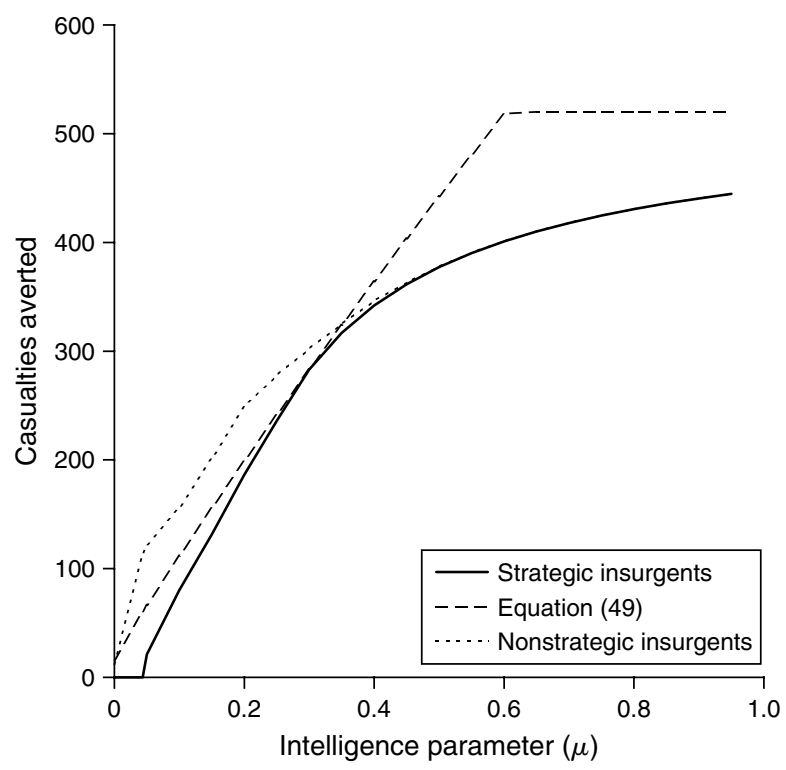

to induce the government to form the knapsack ordering that minimizes casualties averted as discussed in the previous section. The government decides which strongholds to attack by solving Equation (30) and allocating in proportion to those $B_{i}\left(\tilde{y}_{i}\right) \mathrm{s}$ in the optimal battle set.

Figure 3 presents the results. In contrast to the government's optimal ordering from Table 2 (which was Town 1, Town 2, Village 2, Village 1, and Village 3), the insurgents find it optimal to force the government to fight in the larger towns (sequence by decreasing $B_{i}\left(\tilde{y}_{i}\right)$ ) when intelligence is very limited $(\mu<0.15)$. For intermediate values of the intelligence parameter $(0.15<\mu<0.5)$, the insurgents are able to reduce the number of casualties the government can avert by reordering the sequence according to increasing $B_{i}\left(\tilde{y}_{i}\right)$. These strategic responses result in fewer casualties averted when compared to the original towns and villages example with nonstrategic insurgents (when $\mu \geqslant 0.5, f \geqslant$ $\sum_{j=1}^{m} B_{j}\left(\tilde{y}_{j}\right)$ and the government can engage the insurgents in all strongholds). The insurgents' first-mover advantage can be considerable: When $\mu=0.3$, the number of casualties the government can avert falls from 302 to 283; when $\mu=0.2$, the drop is much larger-from 249 to 187 casualties averted, a 25\% reduction. The analytical bound of Equation (49) is also shown in Figure 3. This example shows how smart insurgents can readily create worst-case results for the government, and not surprisingly, the damage inflicted is greater when the government's intelligence is poor.

\section{Incorporating Collateral Civilian Casualties}

Thus far, we have only considered Blue civilian casualties inflicted by the insurgents as modeled by the $k_{i} \mathrm{~s}$, but in counterterror/counterinsurgency operations, governments 
clearly worry about collateral Red civilian casualties even while insurgents/terrorists do not. For example, in an empirical study covering 2001-2003, Kaplan et al. (2005) show that Hamas and other terrorist organizations retaliated with suicide bombing attacks following successful Israeli targeted killings of terror operatives, yet did nothing in response to botched hits that missed their intended targets but resulted in the deaths of Palestinian civilians. We will therefore incorporate Red civilian casualties into the government's damage function while leaving the insurgents' valuation unchanged in a manner similar to Jacobson and Kaplan (2007). Specifically, if the government defeats a stronghold containing $y$ insurgents, Red civilian casualties will be accounted for by the addition of the term $c y$ to the government's damage function. The constant $c$ reflects two assumptions: that the number of Red civilian casualties will be proportional to the number of insurgent casualties, and that the government can differentially value Red versus Blue civilian casualties. Thus, $c$ represents the government's valuation of the number of Red civilian casualties that occur per insurgent casualty. Modeling Red civilian casualties as proportional to insurgent casualties reflects the tendency of insurgents and terrorists to hide in and mix with their civilian population. Even in the case of perfect intelligence, one should expect Red collateral civilian casualties - for example, a rocket-firing crew could be shooting deliberately from the roof of a civilian home still occupied by its family; aimed Blue fire could well harm the family while eliminating the rocket threat. It is reasonable to expect that $c$ would increase as the quality of intelligence deteriorates, that is, there would be more Red civilian casualties in attacks on strongholds where the government has lower levels of intelligence.

If the government has a force of size $x$ with which to attack a stronghold containing $y$ insurgents capable of inflicting $k$ Blue civilian casualties, then the government would only consider attacking if its valuation of the Blue battle and Red civilian casualties caused by attacking is less than its valuation of Blue civilian casualties that would be suffered in the absence of an attack, that is, if

$x-\sqrt{x^{2}-B(y)^{2}}+c y<k$.

Note that if $c y>k$, the government will not attack, irrespective of the magnitude of $x$ and $y$. This introduces a new feature to the analysis: If potential Red civilian casualties are too large, the stronghold is viewed as out of bounds in that no attack there would be approved.

\subsection{Optimal Force Allocation with Collateral Civilian Casualties}

Incorporating Red civilian casualties requires only minor modifications to the force allocation models discussed in $\S 3$. Assume as before that the insurgents have positioned $y_{i}$ fighters and allocated weaponry capable of inflicting $k_{i}$ Blue civilian casualties in stronghold $i$ for $i=1,2, \ldots, m$, and assume further that if the government attacks and conquers stronghold $i, c_{i} y_{i}$ Red civilian casualties will result. Note that allowing the Red civilian casualty rates $c_{i}$ to be stronghold-specific accounts for differential strongholdspecific government intelligence in the model. The government first asks whether $c_{i} y_{i}>k_{i}$ for each stronghold, and for any stronghold where this is the case sets $x_{i}=$ 0 because such strongholds are out of bounds. The government then allocates its $f$ soldiers over the remaining strongholds that are in-bounds in accord with the methods of $\$ 3$ after resetting $k_{i} \leftarrow k_{i}-c_{i} y_{i}$, and the total casualties suffered by the government equal the sum of battle plus true Blue civilian casualties (the original $k_{i} \mathrm{~s}$ ) over inbounds strongholds plus the sum of Blue civilian casualties emanating from those strongholds that are out of bounds. Note that as a result of incorporating Red civilian casualties, the total casualties suffered by the government (the sum of Blue civilian and government battle casualties) can only increase or stay the same. That this is the case for any insurgent deployment across strongholds suggests that the government's desire to avoid Red civilian casualties provides the insurgents with an additional strategic tool, as we explore in the next section.

\subsection{Force Allocation Game with Perfect Intelligence and Collateral Civilian Casualties}

Suppose that the government has perfect intelligence in all strongholds. Recall from Equation (37) that if the insurgents allocate $y_{i}$ fighters to stronghold $i$ in this case, then the battle threshold $B_{i}\left(y_{i}\right)=\sqrt{\beta_{i} / \alpha_{i}} y_{i}$ and government battle casualties resulting from the allocation of $x_{i}$ soldiers to the $i$ th stronghold follow the convex function $x_{i}-\sqrt{x_{i}^{2}-B_{i}^{2}\left(y_{i}\right)}$ provided $x_{i}>B_{i}\left(y_{i}\right)$ (which is a necessary condition for attack). We reconsider the sequential force allocation game where the insurgents first allocate their $\psi$ fighters and weaponry capable of inflicting $\kappa$ Blue civilian casualties across the $m$ strongholds, after which the government allocates its $f$ soldiers. The insurgent goal remains the maximization of Blue battle and civilian casualties, whereas the government objective is now the minimization of Blue battle plus Blue and Red civilian casualties. Let $i^{*}=\arg \max \left(\beta_{i} / \alpha_{i}\right)$ and $i^{* *}=\arg \max \left(c_{i}\right)$. Expanding Proposition 3, we obtain the following result.

Proposition 5. Suppose that the government has perfect intelligence. Then, there are three possible scenarios:

(i) $f \leqslant B_{i^{*}}(\psi)$. Then, the insurgents set $y_{i^{*}}=\psi, k_{i^{*}}=\kappa$, the government does not attack, and the insurgents inflict $\kappa$ Blue civilian casualties.

(ii) $f>B_{i^{*}}(\psi)$ and $\exists i$ such that $f-\sqrt{f^{2}-B_{i}(\psi)^{2}}+$ $c_{i} \psi \geqslant \kappa$. Then, the insurgents set $y_{i}=\psi$ and $k_{i}=\kappa$ for some such $i$, the government does not attack, and once more the insurgents inflict $\kappa$ Blue civilian casualties.

(iii) $f>B_{i^{*}}(\psi)$ and $f-\sqrt{f^{2}-B_{i}(\psi)^{2}}+c_{i} \psi<\kappa \forall i$. Then, if $f-\sqrt{f^{2}-B_{i^{*}}(\psi)^{2}}+c_{i^{*}} \psi>f-\sqrt{f^{2}-B_{i^{* *}}(\psi)^{2}}+c_{i^{* *}} \psi$, the insurgents set $y_{i^{*}}=\psi$ and $k_{i^{*}}=f-\sqrt{f^{2}-B_{i^{*}}(\psi)^{2}}+$ 
$c_{i^{*}} \psi-\epsilon$, for $\epsilon>0$ arbitrarily small, and the government sits and accepts $f-\sqrt{f^{2}-B_{i^{*}}(\psi)^{2}}+c_{i^{*}} \psi-\epsilon$ Blue civilian casualties.

Otherwise, the insurgents put $y_{i^{* *}}=\psi$ and $k_{i^{* *}}=f-$ $\sqrt{f^{2}-B_{i^{* *}}(\psi)^{2}}+c_{i^{* *}} \psi-\epsilon$, the government does not fight, and takes $f-\sqrt{f^{2}-B_{i^{* *}}(\psi)^{2}}+c_{i^{* *}} \psi-\epsilon$ Blue civilian casualties.

Compared to the perfect intelligence game where the government ignores Red civilian casualties, the insurgents' situation has improved in cases (ii) and (iii). Case (ii) shows how the insurgents can take advantage of the government's desire to avoid Red civilian casualties to create out-of-bounds strongholds that allow the infliction of maximal Blue civilian casualties $(\kappa)$, whereas in case (iii) the insurgents are able to guarantee inflicting $\max \left\{f-\sqrt{f^{2}-B_{i^{*}}(\psi)^{2}}+c_{i^{*}} \psi, f-\sqrt{f^{2}-B_{i^{* *}}(\psi)^{2}}+\right.$ $\left.c_{i * *} \psi\right\}$ Blue civilian casualties in circumstances where they would otherwise have had to settle for a smaller number of Blue battle casualties. In all of these cases, even though the insurgents' objective function does not consider Red civilian casualties at all, the knowledge that the government seeks to avoid Red civilian casualties enables the insurgents to gain a strategic advantage. By deliberately blending in with their civilian populations in a manner that would maximize Red civilian casualties if the government attacks, the insurgents can increase the damage they cause to the government. Whether in Lebanon, Gaza, Iraq, or Afghanistan, this is an observable tactic used by terrorists and insurgents the world over.

\section{Conclusions}

We have investigated an important tactical question in counterinsurgency operations: How should the government optimally allocate its forces against insurgent strongholds that threaten civilian populations when both military and civilian casualties must be taken into account, and when the operations are executed with imperfect intelligence? We showed that for a given allocation of insurgents across strongholds, optimally selecting those strongholds to attack can be (approximately) accomplished with a simple knapsack rule. The forces allocated to those strongholds attacked divide the total force available in proportion to the victory thresholds $B_{i}$. These thresholds are determined in turn by the size of the insurgent force in stronghold $i$, the effective fire ratio $\beta_{i} / \alpha_{i}$, and the intelligence function $p(\cdot)$. We also showed that if the insurgents anticipate government actions and have full knowledge of the battle parameters, a sequential game ensues. The solution to this game depends on the government's level of intelligence: If the government has perfect intelligence (and the insurgents know it), then the insurgents' best strategy is to concentrate its entire force and assets in one stronghold, in which case the government decides whether or not to attack depending upon the number of soldiers needed to prevent civilian casualties. With lower levels of intelligence, if the government follows a knapsack policy (which is shown to be nearly optimal), then the insurgents' optimal strategy is to spread their force and materiel in a manner that maximizes the number of soldiers the government needs to win all battles, and induce the government to select battles in a sequence that forces worst-case results. Via example, we showed that the insurgents' first-mover advantage can appreciably reduce the ability of the government to avert both civilian and battle casualties, and this problem is especially acute when the government has poor intelligence. Incorporating Red civilian casualties provides the insurgents with an additional tool, because by mixing with the civilian population, the insurgents can sometimes lead the government to declare strongholds to be out of bounds, resulting in a free pass for the insurgents to attack Blue civilians from such strongholds. This work thus joins other recent research in recognizing the importance of treating our adversaries as strategic players in homeland security, counterterror, or counterinsurgency games (Brown et al. 2006, Golany et al. 2009, Jacobson and Kaplan 2007, Sandler and Arce 2007, Zhuang and Bier 2007).

\section{Electronic Companion}

An electronic companion to this paper is available as part of the online version that can be found at http://or.journal. informs.org/.

\section{Endnotes}

1. The model can be modified to account for limited endurance on both sides, meaning that the combatants are only willing to endure a certain number of casualties before abandoning the fight.

2. A simple extension of the argument to follow applies if civilian or other downstream casualties are valued differently relative to casualties among the attacking government force.

\section{Acknowledgments}

The second and third authors acknowledge support from the Office of Naval Research. The first author acknowledges the Daniel Rose Fund supporting the Technion-Yale Initiative in Homeland Security and Counterterror Operations Research.

\section{References}

Allen, P. D. 1993. Air combat model engagement and attrition processes high level design. RAND Corporation, Santa Monica, CA.

Barno, D. W. 2007. Fighting the "other war": Counterinsurgency strategy in Afghanistan, 2003-2005. Military Rev. 87(September-October) 32-44.

Blanford, N. 2006. Hizbullah and the IDF: Accepting new realities along the Blue Line. MIT Elec. J. Middle East Stud. 6 68-75.

Brown, G., M. Carlyle, J. Salmerón, K. Wood. 2006. Defending critical infrastructure. Interfaces 36(6) 530-544. 
Cordesman, A. H. 2007. Lessons of the 2006 Israeli-Hezbollah War. Center for Strategic and International Studies, Washington, DC.

Cordesman, A. H., E. R. Davies. 2007. Iraq's Insurgency and the Road to Civil Conflict. Praeger Security International, Greenwood Publishing Group, Westport, CT.

Deitchman, S. J. 1962. Lanchester model of guerrilla warfare. Oper. Res. $10818-827$.

Golany, B., E. H. Kaplan, A. Marmur, U. G. Rothblum. 2009. Nature plays with dice-Terrorists do not: Allocating resources to counter strategic versus probabilistic risks. Eur. J. Oper. Res. 192 198-208.

Google Earth. 2008. March. http://earth.google.com.

Hoffman, B. 2004. Insurgency and counterinsurgency in Iraq. OP-127IPC/CMEPP, RAND Corporation, Santa Monica, CA.

Jacobson, D., E. H. Kaplan. 2007. Suicide bombings and targeted killings in (counter) terror games. J. Conflict Resolution 51 772-792.

Kaplan, E. H., A. Mintz, S. Mishal, C. Samban. 2005. What happened to suicide bombings in Israel? Insights from a terror stock model. Stud. Conflict Terrorism 28 225-235.

Kress, M., R. Szechtman. 2009. Why defeating insurgencies is hard: The effect of intelligence in counterinsurgency operations-A best case scenario. Oper. Res. 57(3) 578-585.
Lanchester, F. W. 1916. Aircraft in Warfare: The Dawn of the Fourth Arm. Constable, London.

Morse, P. M., G. E. Kimball. 1946. Methods of operations research. Office of the CNO, Washington, DC.

Rabasa, A., P. Chalk. 2001. Colombian labyrinth: The synergy of drugs and insurgency and its implications for regional stability. RAND Corporation, Santa Monica, CA.

Sandler, T., D. G. Arce. 2007. Terrorism: A game-theoretic approach T. Sandler, K. Hartley, eds. Handbook of Defense Economics. Defense in a Globalized World, Vol. 2. North-Holland, Amsterdam, 775-814.

Schaffer, M. B. 1968. Lanchester models of guerrilla engagement. Oper Res. 16 457-488.

Schreiber, T. S. 1964. Note on the combat value of intelligence and command and control systems. Oper. Res. 12 507-510.

Shelah, O., Y. Limor. 2007. Captive in Lebanon: The Truth About the Second Lebanon War. Miskal-Yedioth Ahrononth and Chemed Books, Tel Aviv (in Hebrew).

Zhuang, J., V. M. Bier. 2007. Balancing terrorism and natural disastersDefensive strategy with endogenous attacker effort. Oper. Res. 55 976-991. 